Journal of

Ecology 1998,

86, 983-988

\title{
Separating the effects of number of individuals sampled and competition on species diversity: an experimental and analytic approach
}

\author{
DEBORAH E. GOLDBERG* and GEORGE F. ESTABROOK \\ Department of Biology, University of Michigan, Ann Arbor, MI 48109-1048, USA
}

\begin{abstract}
Summary
1 Species richness typically increases with the number of individuals sampled, although many ecological processes that influence species richness are also well known to depend on density of individuals. We separated the effects of density on species richness that are due to sampling, from those due to density-dependent ecological processes such as competition or predation, by manipulating the density of an entire community.

2 A seed bank from a community of desert annual plants that occur on semi-stabilized sand dunes in Israel was collected from the field and sown in an experimental garden at a range of densities from 1/16 to eight times the natural density. The species pool observed in the lowest density plots was used as the null community, which was repeatedly sampled to calculate the species richness (and other diversity indices) in higher density plots that would be expected from sampling considerations alone. The significance of deviations of observed diversity from this expected diversity was then evaluated.

3 Both observed and expected number of species increased substantially with the experimental increase in density. However, observed species richness, the ShannonWiener diversity index and Simpson's diversity index were often significantly lower than that expected based on sampling considerations. The magnitude of the deviation from expected increased significantly with increasing density for richness and the Shannon-Wiener index. This provides some of the first direct experimental evidence from diverse natural assemblages that increasing competition among all the individuals in a community can lead to competitive exclusion.
\end{abstract}

Keywords: competition, null community, species-area curves, species diversity, species richness

Journal of Ecology (1998) 86, 983-988

\section{Introduction}

It has long been recognized that the number of species increases with the number of individuals sampled (Fisher etal. 1943; Sanders 1968). This is, in fact, often invoked as one hypothesis to explain the positive slope of a species-area curve (Connor \& McCoy 1979; McGuinness 1984a). However, the implications of this sampling effect for documenting patterns of species richness, and particularly for testing explanations of these patterns, have not been fully explored. [In this paper, we use the term species richness to refer to

(C) 1998 British Ecological Society
Correspondence: Deborah E. Goldberg (fax 734647 0884; e-maildegold@umich.edu). the number of species per unit area, although this is more properly referred to as species density. We do this to avoid any ambiguity that might otherwise result when discussing the relationship between number of species per unit area (species density) and number of individuals per unit area (density)].

The basic problem is that any ecological process that has been postulated to affect species richness directly can also affect density and, thus, indirectly affect richness through the number of individuals sampled. This is perhaps most obvious for competitive interactions: if all else is equal, increasing density will increase the numbers of individuals sampled per unit area and thus increase species richness per unit area. However, the increasing density of indi- 

relationships

(C) 1998 British Ecological Society, Journal of Ecology, 86, 983-988 viduals will also increase the potential for interactions among individuals and thus lead to the loss of species through competitive exclusion. In this case, the sampling effect and the hypothesized ecological effect work against each other such that the two effects could even cancel each other out. If so, an experiment that simply increased density without taking into account the richness-density relationship could conclude that competition has no effect on richness. This conclusion would clearly be false: in the absence of competitive interactions, richness should have increased in the high-density treatment. Similar arguments could be made for the effects of disturbance or predation because these would both be expected to reduce density and therefore would reduce richness based simply on sampling considerations.

Observational studies of patterns of species richness have occasionally taken account of sampling effects due to density (for a cogent history of some of these approaches and examples see Gotelli \& Graves 1996). It has been less common to incorporate sampling effects due to density when developing theoretical explanations for patterns in species richness: the notable exception here has been the recent debate about the cause of unimodal relationships of plant species richness with productivity (Goldberg \& Miller 1990; Abrams 1995; Oksanen 1996; Stevens \& Carson 1996; Grime 1997). However, with the single exception of McGuinness's (1984b) work on the intermediate disturbance hypothesis, we are not aware of any experimental studies of the influence of ecological processes on species richness that have accounted for density-richness relationships. In this paper, we describe a combined experimental and analytical approach for testing the effect of competition on species richness and diversity that takes into account sampling effects on richness that are due to density. The general approach could also be applied to testing effects of predation, disturbance or other processes on species diversity. We illustrate the approach using data from experimental manipulations of density of desert annual communities.

\section{Methods}

\section{GENERAL APPROACH}

The approach used (Fig. 1) involved experimental manipulation of the density of an entire community without altering initial relative abundances of species. The underlying assumption was that increasing initial density increases the potential for interactions among individuals and thus reflects potential competition intensity (Goldberg et al. 1995). A 'null community', i.e. the expected species composition if species are not differentially affected by interactions, is derived from the lowest density plots. A computer program is used to take repeated samples from this null community and generate the expected values of probability dis- tributions of the number of species (or other diversity indices) for plots with different total densities (the solid curve in Fig. 1). These predictions are then compared with the experimentally observed values. The biological null hypothesis is that species richness at the end of an experiment will vary solely as a function of the variation in initial density, i.e. only sampling influences richness (quantity $a$ in Fig. 1). This is equivalent to the statistical null hypothesis that the probability that a given individual belongs to a particular species is invariant among all plots, i.e. it is independent of density or any other parameter.

Competitive interactions could modify the effect of higher density in two distinct ways, both involving an increase in mortality at higher density (or decrease in birth rate) and therefore leading to a final density that is lower than the initial density (Fig. 1). First, to the extent that such density-dependent mortality is randomly allocated among species, richness will decrease solely due to the decrease in number of individuals sampled at the end of the experiment (quantity $b$ in Fig. 1). Secondly, if this mortality falls differentially on initially rare species, richness will be further decreased relative to that expected based on sampling alone (quantity $c$ in Fig. 1). If, however, competitive mortality falls differentially on initially very common species, richness might actually be increased relative to that expected based on sampling alone (not shown in Fig. 1). Thus, increased diversity at higher density, even after taking into account sampling effects, will not necessarily reflect facilitation at the individual level. The net change in final richness between plots with low and high density (quantity $d$ in Fig. 1) reflects the balance of increases due to sampling and changes due to density-dependent mortality or fecundity. It is therefore impossible to quantify either kind of competitive effect or even detect that it exists from such data, unless sampling effects are also considered.

\section{EXPERIMENTAL SYSTEM}

The experimental system was an annual plant community occurring on semi-stabilized sand dunes in the Negev Desert in Israel. The combination of annual life history and the sandy substrate made it possible to collect a community seed bank and to concentrate it by sieving. This concentrated seed bank was then thoroughly mixed to reduce seed aggregations and planted in an experimental garden in eight density treatments: $1 / 16,1 / 8,1 / 4,1 / 2,1,2,4$ and 8 times the natural density of the seed bank. Because greater variation was expected in the lower density plots, the two lowest densities were replicated four times, while all higher densities were replicated twice, for a total of 20 plots. The density treatments up to and including natural density were planted in $1-\mathrm{m}^{2}$ plots. However, because of the labour involved in collecting and sieving the seed bank, the higher density treatments were planted in smaller plots $\left(0.25 \mathrm{~m}^{2}\right)$ to reduce the total 
985

D.E. Goldberg \& G. Estabrook

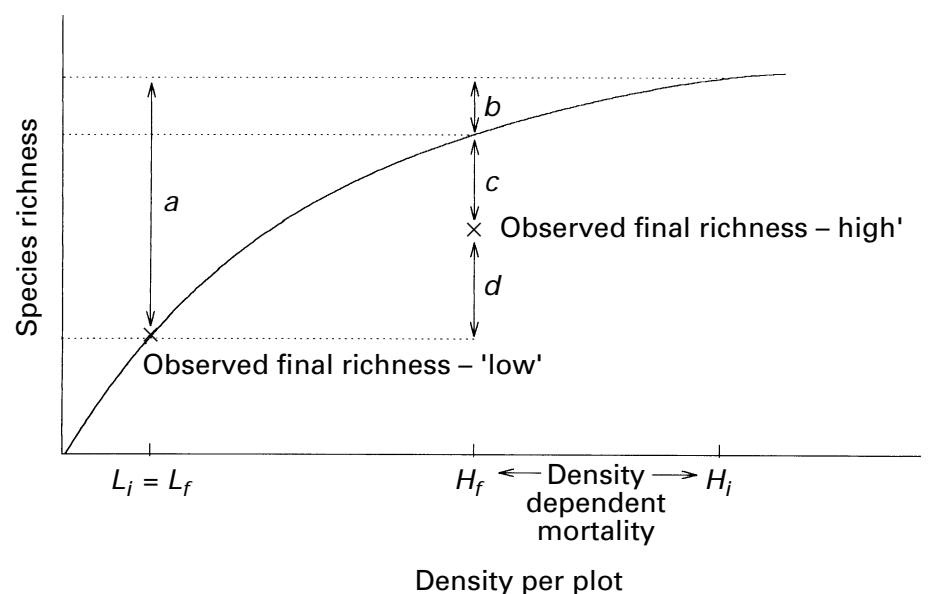

Fig. 1 Effects of numbers of individuals sampled and competitive interactions on species richness. $L_{i}$ and $H_{i}$ represent initial densities for low and high competition intensity, respectively, and $L_{f}$ and $H_{f}$ represent final densities for low and high competition intensities, respectively. In this example, $L_{i}=L_{f}$, while $H_{f}<H_{i}$, indicating that mortality was density dependent. Quantity $a$ represents the change in species richness between low and high competition treatments, which would be expected due to the greater number of individuals sampled at high density. Quantity $b$ represents the decrease in species richness at high competition due only to randomly allocated density-dependent mortality. Quantity $c$ represents the decrease in species richness at high competition due to non-randomly allocated density-dependent mortality. Quantity $d$ represents the net difference in final species richness between low- and high-density plots and is the sum of quantities $a, b$ and $c$, where quantity $a$ is typically positive, quantity $b$ is typically negative, and the sign of quantity $c$ depends on whether density-dependent mortality falls primarily on rare or on common species (see text).

amount of seed bank needed (subsequent experiments in progress use large plots regardless of density). The experimental communities were planted in January 1993, shortly after the winter rainy season had commenced, and harvested in April 1993 at the end of the growing season. Only plants within the central $80 \times 80 \mathrm{~cm}$ area $\left(1-\mathrm{m}^{2}\right.$ plots $)$ or $30 \times 30 \mathrm{~cm}$ area $(0.25-$ $\mathrm{m}^{2}$ plots) were harvested, sorted to species, counted, dried and weighed. The experiment reported here is part of a larger project investigating the effects of productivity on the community-level consequences of competition and the relationship between individual and community-level responses to competition (D. E. Goldberg, R. Turkington \& L. Olsvig-Whittaker, unpublished data).

We characterized the null community by two different species pools. In both cases, only plants surviving to the end of the growing season were included because it was impossible to identify all plants to species at the initial germination phase. We therefore did not have accurate measures of species richness as a function of initial density and so could not quantify effects of randomly allocated density-dependent mortality on species richness (quantity $b$ in Fig. 1). However, there was very little density-dependent mortality overall in this experiment and it is therefore likely that effects of randomly allocated densitydependent mortality on richness were weak, even if present.

The first null community initially only used the $1 / 16$ of the natural density and four at $1 / 8$ of the natural density) as the species pool from which to draw individuals at random. This is biologically the most appropriate species pool to use because it should exhibit minimal density-dependent effects on germination or mortality. However, the total number of individuals in these plots was relatively small (420 vs. 3953 individuals summed over all densities) and thus might, by chance, have non-representative relative abundances. In addition, some relatively rare species in the high-density communities were not present at all in the low-density (no-interaction) communities. To make more plausible predicted distributions possible, we therefore added a single individual of all such species (20 out of 53 total species found in the 20 experimental plots) to this species pool.

In the second species pool, we included all surviving plants in the experiment, regardless of the initial density of the plot. This got around the problems created by the small number of individuals in the low-density plots. On the other hand, this species pool is likely to be biased by species-specific densitydependent germination or mortality at the higher densities. Thus, detection of systematically stronger deviations from expected species composition or diversity at high relative to low density should be strong evidence that, despite this bias, increasing interactions at higher density do affect community structure.

The proportion of individuals belonging to each species in the null community was used as its hypothesized 'invariant probability', i.e. its abundance expected under the hypothesis of no effect of interactions on species richness. These probabilities were used to generate a set of 10000 simulated plots for each sample plot, each having the same total number 

relationships
(C) 1998 British Ecological Society, Journal of Ecology, 86, 983-988 of individuals as its corresponding observed sample plot. For each simulated plot, the species identity of each individual was assigned independently, using the hypothesized invariant probabilities, and these species' identities were used to calculate species richness and the Shannon-Weiner and Simpson diversity indices. The distributions of richness and diversity values from the set of simulated plots were then compared to the corresponding observed value. For each sample plot, the fraction of simulated values greater than or equal to an observed value is referred to as its 'realized high significance', i.e. the probability that diversity at least as high as the observed value could be found by random sampling from the null community. Similarly, the fraction of simulated values less than or equal to each observed value is referred to as its 'realized low significance', i.e. the probability that diversity as low or lower than the observed value could be found by random sampling from the null community. The expected value for a given index at a particular density is estimated by the sum of all the simulated values at that density divided by the number of simulations. The deviations of observed values from these estimated expected values indicate the magnitude of any effects of density on richness and diversity, and the realized significances provide statistical bases for interpreting these magnitudes.

To make the magnitudes of deviations of observed values $(\mathrm{O})$ from estimated expected values $(\mathrm{E})$ more intuitively comparable, we also expressed them in units of expected value $(\mathrm{O}-\mathrm{E}) / \mathrm{E}$. This index gives increasingly negative values as the observed value becomes increasingly smaller than the estimated expected value. This rescaling does not alter the statistical significance of an observed value.

A computer program, DIVDEN (diversity-density analysis), to carry out these analyses, available in Windows 3.1 or DOS versions, can be downloaded from the Journal of Ecology archive on the World Wide Web (for address, see cover of a recent issue).

\section{Results}

The two methods of determining the null community gave qualitatively identical results. We therefore only present results for the species pool derived from the low-density plots, where interactions among individuals are expected to be minimal. The importance of incorporating sampling effects in estimates of diversity is emphasized by the effects of density on the expected richness under the null hypothesis of no effects of interactions on richness or diversity (Fig. 2). Because increasing density increases the number of individuals sampled, both observed richness (data points) and expected richness (line) at the end of the experiment increase with increasing density (Fig. 2). Therefore, if the differences in numbers of individuals sampled had been ignored when comparing observed richness between density treatments, the results would

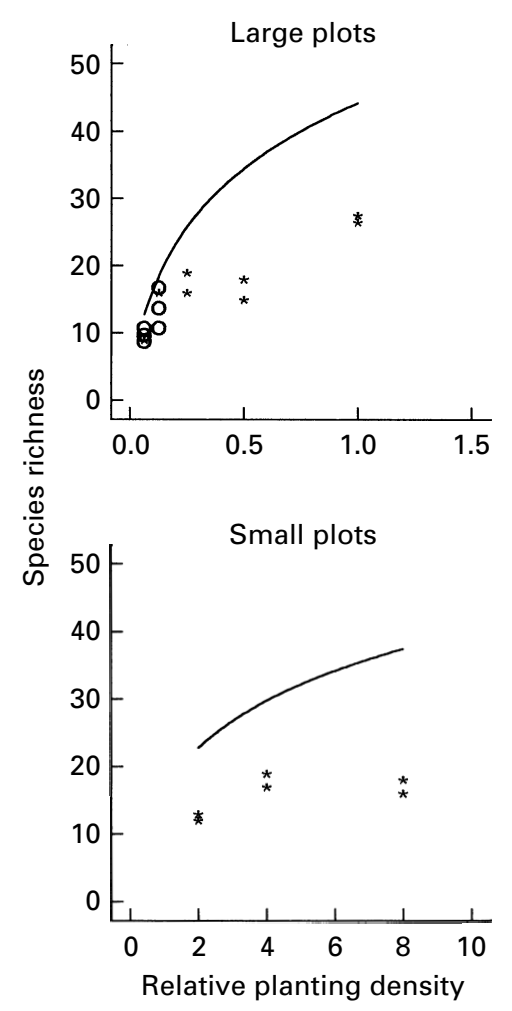

Fig. 2 Observed species richness in plots with different initial planting densities (expressed as density of seed bank relative to natural density). Continuous curved lines pass through expected values of species richness predicted by the hypothesis that interactions do not change the probability that an individual belongs to a particular species. These invariant probabilities are assumed to be proportional to the frequencies observed in the sum of all the lowest density plots ( $1 / 16$ and $1 / 8$ of natural density), modified to include rare species as described in the text. Plots with observed values of species richness significantly $(P<0.05)$ improbable under this hypothesis are designated with * and plots with consistent values are designated with circles. Large plots had a sampled area of $0.64 \mathrm{~m}^{2}$ and included all plots with a planted seed bank density ranging from $1 / 16$ of natural density to natural density. Small plots had a sampled area of $0.09 \mathrm{~m}^{2}$ and included all plots with a planted seed bank density ranging from twice natural density to eight times natural density.

have been taken to indicate facilitation of species richness.

Observed diversity was often less than that expected at a given density under the null. The proportion of significantly lower richnesses (marked with *) was higher at high density (Fig. 2), suggesting that increasing intensity of interactions led to greater competitive exclusion. The reduction in observed species richness relative to that expected under the no-interactions hypothesis increased significantly with increasing potential competition intensity ( $r=-0.50, n=20, P<0.05$; Fig. 3). The same result was observed for the Shannon-Wiener diversity index ( $r=-0.64, n=20, P<0.01)$ and a similar but nonsignificant trend $(r=-0.32, n=20, P=0.16)$ was observed for Simpson's index (Fig. 3). 

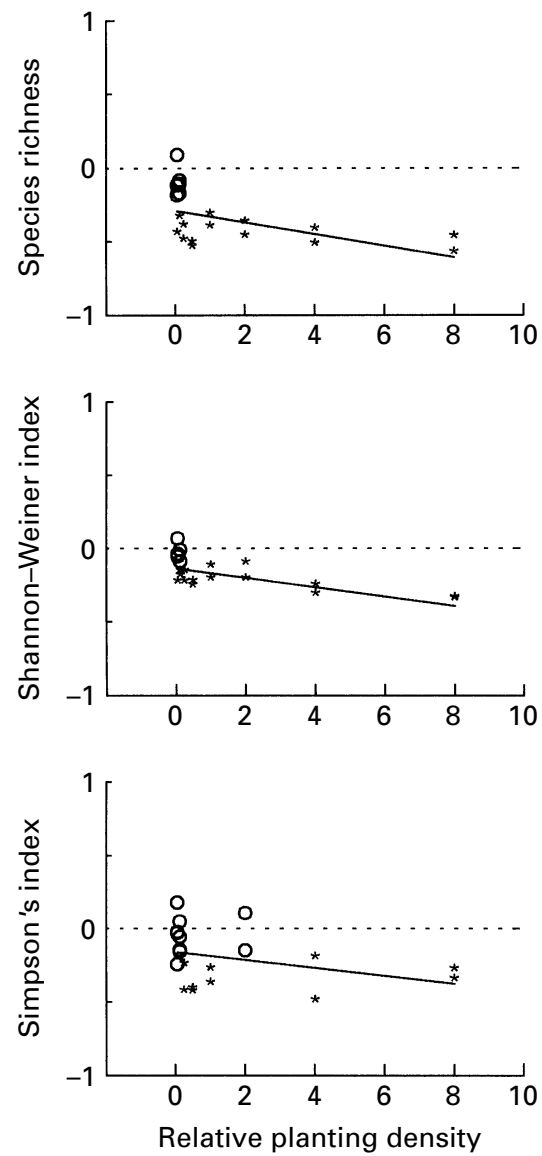

Fig. 3 Deviation of observed from expected measures of diversity expressed in units of expected diversity, in plots with different initial total densities (expressed as density of seed bank relative to natural density). Increasing initial total density is interpreted as increasing intensity of plant-plant interactions. Expected measures are predicted by the hypothesis that interactions do not change the probability that an individual belongs to a particular species. Plots with observed values of species richness significantly $(P<0.05)$ improbable under this hypothesis are designated with * and plots with consistent values are designated with circles. The dashed lines represent no difference between observed and expected and the solid lines are linear regressions through all the points.

\section{Discussion}

In this paper, we have used experimental data to illustrate an approach for separating the effects on species diversity of interactions among individuals from that of the numbers of individuals sampled. The analyses show that increasing intensity of interactions among individuals within a trophic level results in a reduction of species diversity. This result is not surprising, in fact it is almost dogma in ecology. Nevertheless, despite the widespread acceptance of this idea, the effects of overall interaction intensity on diversity have not been tested experimentally previously in real communities with more than two or three species present. The approach illustrated here can also be used to test for effects of interactions on relative abundance of particular species or groups within a community.
Journal of Ecology, 86, 983-988
For example, in the desert annual community, we found that the most abundant species numerically is also the best competitor in the sense that it exhibits the strongest increase in its relative density as density increases (D. E. Goldberg, R. Turkington \& L. Olsvig-Whittaker, unpublished data). However, other common species showed a full range of responses from increases in relative density to strong decreases in relative density as competition became more intense (D. E. Goldberg, R. Turkington \& L. Olsvig-Whittaker, unpublished data).

Regardless of the particular question being addressed, estimating plausible invariant probabilities is essential to the credibility of the results. One difficulty inherent in this estimation is the Narcissus effect where species already completely eliminated due to competition (or lack of facilitation) will not be included and so their observed absence cannot contribute to rejecting the hypothesis that some factor of interest has no effect (Colwell \& Winkler 1984). To the extent that species have already been completely eliminated from the community by plant-plant interactions, the experimental approach illustrated here underestimates the effect of interactions on community structure. On the other hand, the inclusion in the null community of species that are relatively rare but still present in the community ensures that deviations from their predicted abundance in the absence of plant-plant interactions would be detected by this technique if the sample size was large enough.

It is important to note that significant deviations of observed values from the expected values could be due to any factor that causes non-independent observations, not only species interactions. Most notably, any form of intraspecific aggregation (e.g. due to localized dispersal or clonal growth) could result in an observed diversity significantly less than expected. Thus the method illustrated here may be most appropriate for experimental settings where the aggregation of individuals can be largely eliminated. Alternatively, information on aggregation could be included in the calculation of the expected values ( $T$. Rajaniemi, personal communication). In this experiment, we interpret the observed reductions from the expected values of richness and diversity as due to competitive interactions and not due to intraspecific aggregation for two reasons. First, because we thoroughly mixed the seed bank before planting, intraspecific aggregation was minimal in the experimental plots (A. R. Dyer \& D. E. Goldberg, unpublished data). Secondly, the deviations become increasingly negative with increasing density (Fig. 3), while aggregation effects on diversity should be constant, or perhaps even decrease, with increasing number of individuals sampled.

In the example reported here, the importance of adjusting for effects of number of individuals sampled on measures of diversity is obvious because density was directly manipulated and hence deliberately cov- 
Diversity-density relationships ered a very broad range, and other potentially contributing factors were held relatively constant in the experiment. However, accounting for effects of numbers of individuals may be equally important in other kinds of situations where density is not manipulated directly but is likely to change in response to an experimental treatment or environmental characteristic. For example, it is usually assumed in plant ecology that density declines at high fertility because of increases in individual plant size and limitations on total biomass per unit area leading to greater densitydependent mortality (Harper 1977; Tilman \& Pacala 1993). Similarly, the presence of predators, disturbance or a severe physiological stress (e.g. a long drought) may all increase mortality.

It is unclear to what extent patterns in diversity over natural or experimental landscapes can be explained simply by such patterns in numbers of individuals, because most available data on diversity do not account for variation in numbers. These are especially difficult data to obtain for communities dominated by clonal organisms, such as most herbaceous perennial plant communities. Nevertheless, effects of numbers of individuals sampled have been incorporated into several different hypotheses to explain at least one kind of diversity pattern: relationships with productivity (Goldberg \& Miller 1990; Wright et al. 1993; Oksanen 1996; Stevens \& Carson 1996). In one attempt to evaluate the role of density changes in the reduction in richness associated with fertilizer addition, Goldberg \& Miller (1990) found that addition of nitrogen did indeed decrease density and that this decreased number of individuals contributed to, but did not explain completely, the decline in species richness. Similar important effects of number of individuals sampled on diversity patterns seem likely to be general. The combined experimental and analytical approach described here for quantifying the effect of community-wide interactions on species diversity independently of sampling effects opens the way for direct experimental testing of numerous hypotheses about community-level consequences of competition and other species interactions.

\section{Acknowledgements}

We are grateful to Andy Dyer, Lindsay Haddon, Chad Hershock, Tim Howard, Katie Nash, Jari Oksanen, Tara Rajaniemi, Henry Stevens, Roy Turkington, David Ward and an anonymous referee for discussion or critical comments on earlier drafts of the manuscript. We are also grateful to Roy Turkington and Linda Olsvig-Whittaker for their col- laboration in the experiments with D. E. Goldberg. This work was supported by the BiNational Science Foundation US-Israel, the National Science Foundation and the University of Michigan.

\section{References}

Abrams, P.A. (1995) Monotonic or unimodal diversity-productivity gradients: what does competition theory predict? Ecology, 76, 2019-2027.

Colwell, R.K. \& Winkler, D.W. (1984) A null model for null models in biogeography. Ecological Communities: Conceptual Issues and the Evidence (eds D.R. Strong, D. Simberloff, L.A. Abele \& A.B. Thistle), pp. 344-359. Princeton University Press, Princeton, NJ.

Connor, E.F. \& McCoy, E.D. (1979) The statistics and biology of the species-area relationship. The American Naturalist, 113, 791-833.

Fisher, R.A., Corbet, A.S. \& Williams, C.B. (1943) The relation between the number of species and the number of individuals in a random sample of an animal population. Journal of Animal Ecology, 12, 42-58.

Goldberg, D.E. \& Miller, T.E. (1990) Resource additions and species diversity in an annual plant community. Ecology, 71, 213-225.

Goldberg, D.E., Turkington, R. \& Olsvig-Whittaker, L. (1995) Quantifying the community-level effects of competition. Folia Geobotanica and Phytotaxonomica, 30, 231-242.

Gotelli, N.J. \& Graves, G.R. (1996) Null Models in Ecology. Smithsonian Institution Press, Washington, DC.

Grime, J.P. (1997) The hump-backed model: a response to Oksanen. Journal of Ecology, 85, 97-98.

Harper, J.L. (1977) Population Biology of Plants. Academic Press, London, UK.

McGuinness, K.A. (1984a) Equations and explanations in the study of species-area curves. Biological Reviews, 59, 423-440.

McGuinness, K.A. (1984b) Species-area relations of communities on intertidal boulders: testing the null hypothesis. Journal of Biogeography, 11, 439-456

Oksanen, J. (1996) Is the humped relationship between species richness and biomass an artefact due to plot size? Journal of Ecology, 84, 293-295.

Sanders, H.L. (1968) Benthic marine diversity: a comparative study. The American Naturalist, 102, 243-282.

Stevens, M.H.H. \& Carson, W.P. (1996) A theoretical demonstration that the unimodal diversity-productivity curve can be a sampling artefact. Bulletin of the Ecological Society of America, Program and Abstracts Part 2, 77 (Suppl.), 423.

Tilman, D. \& Pacala, S.W. (1993) The maintenance of diversity in plant communities. Species Diversity in Ecological Communities (eds R.E. Ricklefs \& D. Schluter), pp. 1326. University of Chicago Press, Chicago, IL.

Wright, D.H., Currie, D.J. \& Maurer, B.A. (1993) Energy supply and patterns of species richness on local and regional scales. Species Diversity in Ecological Communities (eds R.E. Ricklefs \& D. Schluter), pp. 66-74. University of Chicago Press, Chicago, IL.

Received 6 May 1997

revision accepted 18 May 1998 